\title{
Intermittent Practice Facilitates Stable Motor Memories
}

\author{
Simon A. Overduin, ${ }^{1}$ Andrew G. Richardson, ${ }^{2}$ Courtney E. Lane, ${ }^{1}$ Emilio Bizzi, ${ }^{1}$ and Daniel Z. Press ${ }^{3}$ \\ ${ }^{1}$ Department of Brain and Cognitive Sciences and McGovern Institute for Brain Research, Massachusetts Institute of Technology, Cambridge, \\ Massachusetts 02139, 2Division of Health Sciences and Technology, Massachusetts Institute of Technology and Harvard Medical School, Cambridge, \\ Massachusetts 02142, and ' ${ }^{3}$ Department of Neurology, Beth Israel Deaconess Medical Center and Harvard Medical School, Boston, Massachusetts 02215
}

Humans adaptively control reaching movements to maintain good performance in the presence of novel forces acting on the arm. A recent study suggested that motor memories of different force conditions are not transformed from fragile to stable states, but rather are always vulnerable to interference from newly learned conditions (Caithness et al., 2004). This is contrary to the results of previous studies (Brashers-Krug et al., 1996; Shadmehr and Brashers-Krug, 1997), although all of these studies followed similar methods. Here, we show that a seemingly insignificant and inconsistently applied methodological detail may reconcile this discrepancy. Catch trials, in which the novel forces are removed, may be randomly interspersed among the more frequent force trials to assess how a subject is learning to predict the pattern of forces. In the absence of an interfering condition, subjects retained their learning until retest a day later regardless of whether they experienced catch trials. But in the presence of an interfering condition, only the subjects who had experienced forces intermittently retained their learning and thereby showed resistance to the interference. Thus, intermittent rather than constant practice conditions appear to be critical for dynamic motor memory stabilization.

Key words: motor; movement; learning; consolidation; memory; arm

\section{Introduction}

The memory consolidation hypothesis states that a memory is transformed from a labile to a stable state in a gradual, timedependent manner (McGaugh, 2000). Consolidation in the motor system was originally demonstrated by Shadmehr and coworkers (Brashers-Krug et al., 1996; Shadmehr and BrashersKrug, 1997) using a task in which subjects learned to reach in novel dynamical environments, or "force fields," created by a robotic manipulandum.

To track adaptation to these force fields, the initial studies would intermittently turn the force field off (i.e., present a null field) during random trials referred to as "catch trials." This manipulation was not thought to impact memory consolidation, but was merely used as a tool to assay field-specific learning (Brashers-Krug et al., 1996; Shadmehr and Brashers-Krug, 1997). In a more recent study, Caithness et al. (2004) failed to show consolidation in the force field task, but rather found continued sensitivity to an interfering condition even at $1 \mathrm{~d}$ or more after learning. But Caithness et al. (2004) also did not use catch trials as in the original literature. Instead, they presented the force field relatively continuously during each learning session, assessing specificity of learning with a block of null-field trials only after learning was completed.

Here, we tested whether the presence or absence of catch trials during the course of force field learning could explain the differ-

\footnotetext{
Received March 28, 2006; revised Sept. 12, 2006; accepted 0ct. 10, 2006.

This work was supported by National Institutes of Health Grant K23-MH65434 (D.Z.P.). We thank Martin Ramos Rizo-Patron for engineering assistance.

Correspondence should be addressed to Dr. Daniel Z. Press, Assistant Professor in Neurology, Beth Israel Deaconess Medical Center and Harvard Medical School, 330 Brookline Avenue, Boston, MA 02215. E-mail: dpress@bidmc.harvard.edu.

DOI:10.1523/JNEUROSCI.1320-06.2006

Copyright $\odot 2006$ Society for Neuroscience $\quad 0270-6474 / 06 / 2611888-05 \$ 15.00 / 0$
}

ent outcomes of these studies. In particular, we tested whether subjects' force field learning could survive exposure to an interfering force field and whether such retention varied depending on their experience of catch trials or continuous forces. Our results indicate that subjects practicing with or without such interruptions of the force field do indeed demonstrate markedly different patterns of skill retention.

\section{Materials and Methods \\ Subjects}

This study involved 40 right-handed, English-speaking subjects (mean, 23.0 years old; 21 males). Participants were screened for history of neurological illness, epilepsy, seizures, head injuries, and neurosurgery. Ethical approval was obtained through the Massachusetts Institute of Technology Committee on the Use of Humans as Experimental Subjects. Subjects were required to sleep at least $6 \mathrm{~h}$ in the two nights preceding and the night during the experiment. The 40 participants were assigned to four experimental groups of 10 subjects each in a $2 \times 2$ design, defined by (no or one) interfering epoch between learning and retest epochs and $(0$ or $20 \%$ ) catch trials in all force epochs.

\section{Paradigm}

Task. Subjects held onto a robotic manipulandum and used it to make visually guided reaching movements. During these movements, the forearm was held approximately level by means of a ceiling-mounted sling. The targets were white 1-cm-wide squares appearing on a black background, as displayed on a vertically oriented monitor above the planar manipulandum apparatus. Target distances were $10 \mathrm{~cm}$ both in real space and as shown on the monitor. Motions of the handle were represented on the screen as continuous movements of a $0.8-\mathrm{cm}$-wide white crosshair. The six peripheral targets were spaced hexagonally around a seventh, central target to ensure equidistant separation of neighboring targets. Participants were given $0.5 \pm 0.5$ s to complete each movement, starting from the moment the target was presented and lasting until their cursor entered the target. The target remained on the screen even if subjects failed to reach it in the allotted time. On acquiring the target, an addi- 
tional $0.5 \mathrm{~s}$ within-target hold time had to be observed in order for the trial to be a "success." Trial success was indicated to the subject by a transition in the color of the target from white to green just before its disappearance. If either of the conditions was not met, the trial failure was instead indicated by a target transition from white to red.

Design. Subjects first completed a "baseline" epoch in which no force field was applied, followed 5 min later by a "learning" epoch in which a clockwise velocity-dependent rotational force field was applied. An "interference" epoch with a counterclockwise force field was then presented $6 \mathrm{~h}$ later to two of the groups. Finally, all subjects returned $24 \mathrm{~h}$ after the end of their previous epoch for a "retest" epoch in the clockwise force field. The epochs themselves required $\sim 8 \mathrm{~min}$ to complete. We did not control the activities of the participants in the remaining intervals, except to require that the $24 \mathrm{~h}$ interval include at least $6 \mathrm{~h}$ of sleep (verified by subject report).

Instructions. The basic task was described, and subjects were asked not to "anticipate" the targets but merely to move as "naturally" as possible. They were informed that targets would often be difficult to reach in the allotted time, particularly at the beginning of the experiment. The trial "success" feedback was described, but subjects were told it was given merely to help them make their movements as consistently as possible, and not to evaluate how successful they were. As a safety precaution, participants were warned before each epoch that they might experience "forces" generated by the robot.

Target sequence. Each epoch was defined by a pseudorandom sequence of 216 targets that was the same for all subjects. All participants completed each movement specified by the target sequence; trials were not aborted if they failed to reach the target on time. After each trial and an additional $0.1 \mathrm{~s}$ period, a new target appeared and the former target became the "origin" for the next movement. The first origin in each epoch was always the central square. From each of the six peripheral origins, the possible targets were restricted to one of the three neighboring equidistant targets. From the central origin, movements to only three of the six candidate directions were allowed. The target sequence was constrained to have equal frequencies of both the six movement directions possible from the set of peripheral targets and the three directions allowed from the central target. In addition, we equalized the frequency of each movement origin/target combination as much as possible, by requiring that each target sequence contain at least seven trials in each of these 21 movement path possibilities.

Forces. All subjects experienced a null $(0 \mathrm{~N} \cdot \mathrm{s} / \mathrm{m})$ force field during the baseline epoch, a velocity-dependent clockwise field in the learning and retest epochs, and a counterclockwise field in the interference epoch. The curl forces, of magnitude $10 \mathrm{~N} \cdot \mathrm{s} / \mathrm{m}$, were calculated on-line as

$$
f=\mathbf{B} \dot{x},
$$

where

$$
\mathbf{B}=\left[\begin{array}{cc}
0 & 10 \\
-10 & 0
\end{array}\right]
$$

(clockwise) or

$$
\mathbf{B}=\left[\begin{array}{cc}
0 & -10 \\
10 & 0
\end{array}\right]
$$

(counterclockwise) and $\dot{x}$ was the endpoint (i.e., hand) velocity.

Catch trials. On these trials (experienced by one-half of the subjects), the motors were turned off suddenly, silently, and with no warning to the subject, just as the target was presented. Catch trials occurred on the same trials for both groups of catch trial subjects. These trials were preprogrammed pseudorandomly such that in each epoch, exactly two catch trials occurred among each of the 21 movement paths defined by the allowable origin and target combinations (see above, Target sequence). In addition to these 42 catch trials per epoch, on the "zeroth trial" of each epoch all catch and noncatch trial subjects moved the cursor to the central origin in the absence of forces, to begin the epoch proper. Thus, of the 217 trials including the zeroth trial, 43 (or $\sim 20 \%$ ) were performed without forces by the catch trial subjects. There was no additional constraint that they were to occur at a regular rate within each epoch, but at least one catch trial was present in each 18-trial bin.

\section{Analysis}

Velocity vector correlation. Adopting a traditional method of gauging force field adaptation (Shadmehr and Brashers-Krug, 1997; Caithness et al., 2004), we computed the similarity between subjects' velocity profiles in the force field epochs with their velocities late in the baseline epoch. Velocity data were obtained from manipulandum tachometer signals, translated into endpoint coordinates. Given an $[x, y]$ pair of velocity vectors $\mathbf{n}$ for the null-field baseline epoch and a corresponding vector pair $\mathbf{f}$ for a force field trial, the measure computes the correlation coefficient as follows:

$$
\begin{aligned}
\mathrm{CC}(\mathbf{n}, \mathbf{f})=\frac{\operatorname{cov}(\mathbf{n}, \mathbf{f})}{\sqrt{\operatorname{var}(\mathbf{n}) \cdot \operatorname{var}(\mathbf{f})}} \\
\quad=\frac{\mathrm{E}(\mathbf{n} \cdot \mathbf{f})-\mathrm{E}(\mathbf{n}) \cdot \mathrm{E}(\mathbf{f})}{\sqrt{[\mathrm{E}(\mathbf{n} \cdot \mathbf{n})-\mathrm{E}(\mathbf{n}) \cdot \mathrm{E}(\mathbf{n})][\mathrm{E}(\mathbf{f} \cdot \mathbf{f})-\mathrm{E}(\mathbf{f}) \cdot \mathrm{E}(\mathbf{f})]}} .
\end{aligned}
$$

Following Caithness et al. (2004), we calculated the baseline $x$ and $y$ velocity vectors as the average of the last four baseline trials performed in each of the (21) allowable movement paths. Note that this procedure differs from that of Shadmehr and Brashers-Krug (1997), who did not calculate average baseline speed vectors but rather selected individual baseline speed vectors that were optimally correlated to subsequent vectors. Because a $0.5 \pm 0.5 \mathrm{~s}$ time limit was applied to the sum of subjects' reaction plus movement time, rather than a tighter $0.5 \pm 0.05 \mathrm{~s}$ time limit applied to the movement time alone (Caithness et al., 2004), we adjusted the method to allow for greater variability in the timing and duration of the velocity profiles. Specifically, we aligned the profiles on the time of peak speed rather than the time that a minimum speed threshold was met, and we used $1 \mathrm{~s}$ rather than $0.5 \mathrm{~s}$ velocity vectors (Caithness et al., 2004). In doing so, we averaged together or correlated the vectors only over the portions of the vectors that overlapped one another.

Learning index. An additional measure of performance allowed us to gauge the degree to which catch trial subjects learned to anticipate the forces, rather than react to them by cocontraction (Donchin et al., 2002). This "learning index" was based on the signed (counterclockwise-clockwise) perpendicular displacement (PD) of each movement at $300 \mathrm{~ms}$ after reaction time. Position data were derived from manipulandum joint encoder position signals, translated into endpoint coordinates. Displacement was defined relative to a straight line connecting the centers of the origin and target squares and thereby defining an idealized trajectory. Reaction time was defined as the time the speed profile reached $20 \%$ of its peak on the trial. Several other measures of trajectory deviation (e.g., signed peak perpendicular deviation, deviation angle or area) yielded qualitatively similar results. To summarize the catch trial subjects' internalization of each field, we computed a learning index (LI) that expressed performance in each trial bin as a ratio of PDs on catch trials and all trials:

$$
\mathrm{LI}=\frac{\mathrm{PD}_{\text {catch }}}{\left|\mathrm{PD}_{\text {fielded }}\right|+\left|\mathrm{PD}_{\text {catch }}\right|}
$$

The measure is like that of Donchin et al. (2002), except that we allow the numerator to be signed to make clear the distinction between the two curl fields.

Comparisons. Trials in which the subject failed to reach the target in the allotted $0.5 \pm 0.5 \mathrm{~s}$ time limit were still included in the analysis, unless the reaction time was $<0.2 \mathrm{~s}$ or the combined reaction plus movement time was $>1.5 \mathrm{~s}$. Trials were binned by 18 trials in each epoch and condition. Catch trials were excluded from the trial bins for the CC measure. We do not present data from the baseline epoch because performance, as described in the preceding two sections, was either defined relative to this epoch (CC) or based on catch and fielded trials (LI). Statistical results were based on the within-bin-averaged CC or LI values. The transfer of clockwise field learning across epochs was estimated by comparing the mean scores of the first two 18-trial bins in the retest epoch with the last two trial bins in the learning epoch. Main and interaction effects of between-subjects interference and catch trial frequency and withinsubjects time bin (or bin average, in the case of learning transfer) were assessed using repeated-measures ANOVAs. All significant effects (at the $p<0.01$ level) are reported. 


\section{Results}

Subject trajectories were deviated in the patterns typical of rotational force fields (Fig. 1A). After the introduction of a clockwise force field in the learning epoch, movements from the center to the periphery became deviated in a clockwise direction. Essentially the opposite pattern of errors was evident in movements performed with the counterclockwise field during the interference epoch $6 \mathrm{~h}$ later, but clockwise-deviated movements were again expressed at retest $24 \mathrm{~h}$ later still. The two curl fields were also associated with multimodal velocity profiles (Fig. $1 B)$, particularly in the learning epoch, indicating corrective submovements that became attenuated with practice.

To quantify performance in the task, we computed the velocity vector correlation between force trials (in the learning, interference, retest epochs) and null trials (baseline epoch; see Materials and Methods). Results from the two control groups (with and without catch trials) experienc-

ing no interference are shown in Figure $2 A$, whereas the two groups who did experience an interference condition are shown in Figure $2 B$.

Within the learning epoch, all four groups experienced a comparable increase in performance as measured by the velocity vector correlation. In an interference by catch trial by time bin repeated-measures ANOVA of this epoch, the only significant effect was that of time $\left(F_{(11,396)}=17.72 ; p<0.0001\right)$. Within the interference epoch, both 0 and $20 \%$ catch trial groups demonstrated similar performance in the counterclockwise field, with the only significant effect being that of time $\left(F_{(11,198)}=2.89 ; p=\right.$ $0.0053)$. Within the retest epoch, there was again a significant effect of time across the four groups $\left(F_{(11,396)}=11.11 ; p<\right.$ $0.0001)$, but both the presence of an interfering epoch and the presence of catch trials influenced recall (interference by time: $F_{(11,396)}=3.15, p=0.0016$; interference by catch trial by time: $\left.F_{(11,396)}=3.62, p=0.0003\right)$. The effect of catch trials in the retest epoch was limited to the interference groups (Fig. $2 B$ ): only among these subjects was there a significant catch trial by time interaction $\left(F_{(11,198)}=3.03 ; p=0.0033\right)$, in addition to a significant main effect of time $\left(F_{(11,198)}=10.17 ; p<0.0001\right)$. Noninterference groups (Fig. 2A) only showed the effect of time $\left(F_{(11,198)}=3.76 ; p=0.0002\right)$.

To describe these recall differences in reference to initial clockwise field learning, we compared the average of the final two learning-epoch trial bins with the average of the first two retest bins, for all groups, using an interference by catch trial by bin average ANOVA. The relative retest performance of the group given an interference epoch but no catch trials appeared to be deviant relative to the other groups (Fig. $2 A, B$ ). Indeed, we found significant effects not only of bin average $\left(F_{(1,36)}=21.20 ; p<\right.$ $0.0001)$ but of interference by catch trial by bin average $\left(F_{(1,36)}=\right.$ $6.07 ; p=0.0090)$.

The retest difference between the 0 and $20 \%$ catch trial subjects (Fig. 2 B) did not appear to be attributable to coactivation by the latter subjects. Subjects given $20 \%$ catch trials (with or without an interference epoch) appeared to internalize the clockwise
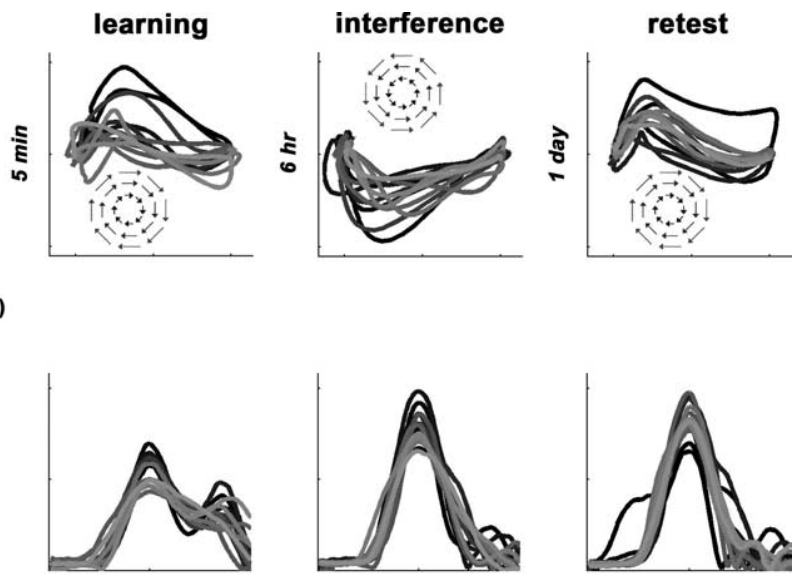

force field in the learning epoch (Fig. 2C), as measured by an increasing LI (i.e., aftereffects on catch trials expressed as a fraction of total, absolute trajectory error; see Materials and Methods). Indeed, an interference by time bin repeated-measures ANOVA revealed a significant effect only of time on the LI $\left(F_{(11,19)}=14.84 ; p<0.0001\right)$. A comparable development of clockwise LI characterized the interference epoch, where a repeated-measures (time bin) ANOVA on the interference subjects alone again revealed a significant effect of time $\left(F_{(11,9)}=\right.$ $5.77 ; p<0.0001)$. In the retest epoch, an interference by time bin ANOVA again found only a significant effect of time $\left(F_{(11,19)}=\right.$ 9.88; $p<0.0001)$. A small difference in the LI initially apparent between the groups given or spared an interfering field (Fig. 2C) may have underlain a nonsignificant trend toward an interference by time interaction $(p=0.0310)$.

\section{Discussion}

Our results indicate that participants who had experienced the force field intermittently, because of the presence of catch trials, appeared uniquely able to retain their initial learning regardless of whether they had subsequently experienced an interfering force field. Catch trials have previously been shown to lead to an undercompensation for forces during initial learning (Thoroughman and Shadmehr, 2000), but their effect on learning retention has not previously been appreciated. In the following discussion, we consider several features of the catch trial subjects' experience that could account for the relative stability of their motor memory: 1) the characteristic error feedback they generated;2) the presence of interruptions of the force field; and 3) the unpredictability of these interruptions.

First, the distinct errors experienced on catch trials might well emphasize the different dynamic conditions experienced on force trials, but how precisely this error feedback impacts learning retention is uncertain. Our paradigm bears resemblance to those used by Wada et al. (2003) and Osu et al. (2004), who alternated two oppositely directed velocity-dependent fields within epochs. These studies found participants could recall each field when the fields were presented with distinct sensory cues. It is conceivable 


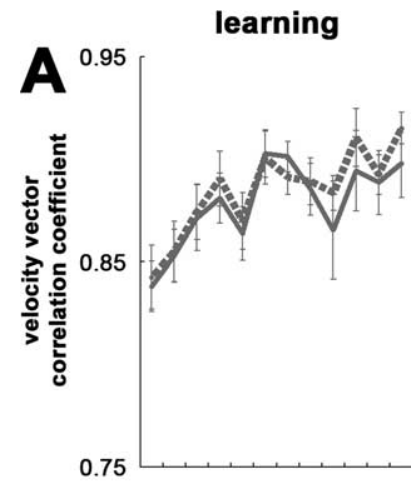

interference
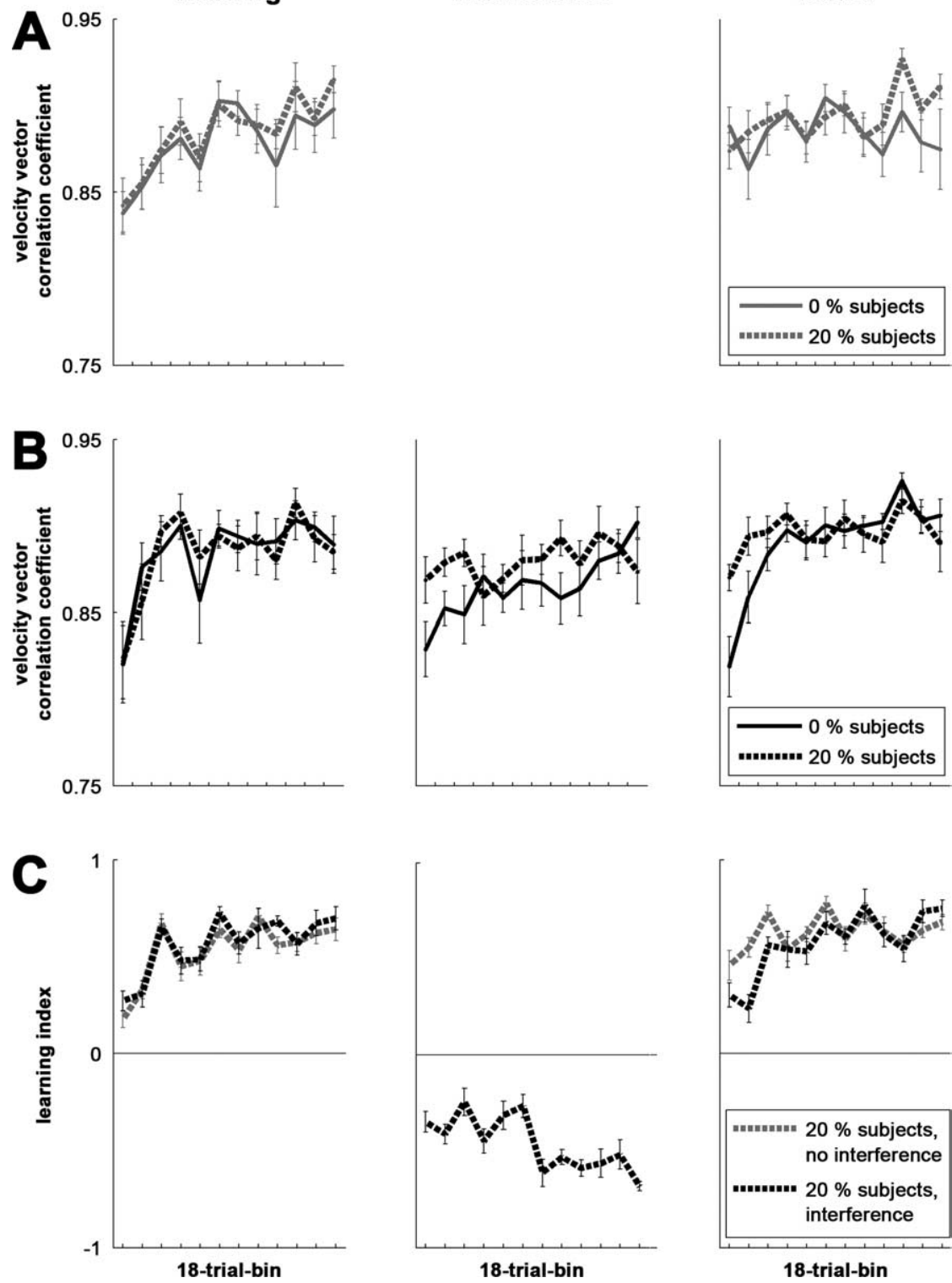

Figure 2. Catch trials are associated with increased stabilization of dynamic motor learning. $A$, Subjects given continuous or intermittent forces (i.e., 0 or $20 \%$ catch trials) in a clockwise epoch, but no subsequent interfering field, begin performing at better than naive levels at retest, according to the velocity vector correlation. $\boldsymbol{B}$, Subjects given an interfering field only exhibited such retention if they had experienced the forces intermittently. $\boldsymbol{C}$, Subjects experiencing intermittent forces internalized the clockwise and counterclockwise fields, as measured by an increase in the learning index (i.e., the relative magnitude of trajectory deviations accounted for by the catch trials). Lines, Mean \pm SEM of trial bins.

that our subjects learned to associate their distinctive visual and proprioceptive error on catch and force trials with the null and curl fields, respectively. Distinct error feedback could also have caused subjects to consciously attend to the perturbations they experienced (Malfait and Ostry, 2004). However, Klassen et al. (2005) found that subjects were able to recall the force field of a previous day regardless of whether they had been introduced to the full force field gain from the first trial of learning or had had the field presented incrementally. This study implies that salient movement feedback errors are not required for the recall of dynamic motor learning, even if they may facilitate cognitively mediated interlimb transfer of the adaptation (Malfait and Ostry, 2004). Our results are consistent with this latter finding insofar as both the 0 and $20 \%$ catch trial subjects were identically able to recall their uninterfered clockwise force field learning from the previous day (Fig. 2A), despite the characteristic aftereffect errors of the $20 \%$ catch trial subjects (Fig. 2C).

Second, the catch trial subjects' apparent memory stabilization did not likely benefit simply from interruptions of the force field. At a cellular level, visual activity-induced synaptic modifications are afforded resistance against subsequent interference from white-noise stimuli by intermittent, rather than continuous, presentation of the initial stimuli (Zhou et al., 2003). In the motor system, our results parallel previous findings showing longterm benefits of distributed rather than massed practice (Schmidt and Lee, 1999). But such interruptions, in and of themselves, may not be sufficient to stabilize motor memories. Research into saccadic adaptation by primates with either a block of catch trials or an equivalent period of rest without visual inputs suggests that actual practice under null conditions is required to cause active unlearning and relearning of the novel condition (Kojima et al., 2004).

Third, this "active" learning, and memory stabilization, may have benefited from the relative unpredictability of the catch trials. Monotonous repetition of a motor behavior leads to superior immediate performance, but practice with some degree of randomness facilitates long-term retention (Shea and Morgan, 1979; Proteau et al., 1994; Osu et al., 2004). Similarly, in operant conditioning experiments, it has long been known that variable reinforcement schedules lead to associations that are more resistant to extinction than those generated by continuous reward regimens (Skinner, 1953). Although the common interpretation is that subjects are more motivated to continue producing a behavior if they have even a slight, probabilistic expectation of reward (Myers, 1998), we suggest that the durability of these associations could also be a result of their greater stabilization in memory.

Note that the unpredictability of the forces experienced by the catch trial subjects did not lead them to use a muscular coactivation strategy, as demonstrated by their pattern of increasing aftereffects on catch trials in each epoch (Fig. 2C). Although cocontraction is a reasonable response when the force field gain on each trial is genuinely unpredictable (Takahashi et al., 2001), we did not expect such a strategy in our experiment, given both that the field was still present on a majority of trials, and that a $1 / 5$ catch trial rate is comparable with the $1 / 6$ rate previously associated with force field internalization (Shadmehr and Brashers-Krug, 1997). However, coactivation by the participants given no catch trials was also possible, and cannot be ruled out because they did not experience the catch trials by which we could gauge such coactivation.

Although the above discussion assumes that intermittent rather than continuous presentation of forces was associated with 
protection from retrograde interference, anterograde interference effects are also possible. Indeed, among catch trial subjects, the time course of their aftereffects appeared to be different in the learning and interference epochs, with prominent aftereffects delayed until about halfway through the latter (Fig. $2 C$ ). This pattern suggests that $6 \mathrm{~h}$ after learning the clockwise field, the catch trial subjects still experienced anterograde interference (Miall et al., 2004) when acquiring the counterclockwise field. At retest, these subjects might have experienced additional anterograde interference, given their initially lower, if statistically similar, performance relative to noninterference subjects (Fig. 2C). Such anterograde interference has been observed previously even $24 \mathrm{~h}$ after an interfering condition (Shadmehr et al., 1998; Donchin et al., 2002). It is possible that the $0 \%$ catch trial subjects' relatively poor recall after interference (Fig. $2 B$ ) could have been attributable to greater anterograde interference at retest relative to the $20 \%$ catch trial subjects, rather than greater retrograde interference (Miall et al., 2004).

Whatever the relationship between intermittent practice and motor memory stabilization, and whatever the direction of interference between epochs, our empirical findings at least suggest that subjects given uninterrupted force fields were uniquely susceptible to an interfering field, even at $6 \mathrm{~h}$ after learning. The presence or absence of catch trials during force field learning can thus reconcile the differences between Brashers-Krug et al. (1996) and Caithness et al. (2004). This is the case although our paradigm is a distinctly more generalized version of the standard center-out reaching task, with more variability in movement paths and their ordering, and in movement speed. As such, our paradigm provided a test for the generality, as well as the validity, of the original motor consolidation result (Brashers-Krug et al., 1996).

Although our experiment was designed to test the stability of motor memories, it did not explore the second aspect of consolidation theory, namely the time course of stabilization. Additional work, with careful attention to methodological detail, will be needed to probe determinants of this time course (Krakauer et al., 2005).

\section{References}

Brashers-Krug T, Shadmehr R, Bizzi E (1996) Consolidation in human motor memory. Nature 382:252-255.

Caithness G, Osu R, Bays P, Chase H, Klassen J, Kawato M, Wolpert DM,
Flanagan JR (2004) Failure to consolidate the consolidation theory of learning for sensorimotor adaptation tasks. J Neurosci 24:8662-8671.

Donchin O, Sawaki L, Madupu G, Cohen LG, Shadmehr R (2002) Mechanisms influencing acquisition and recall of motor memories. J Neurophysiol 88:2114-2123.

Klassen J, Tong C, Flanagan JR (2005) Learning and recall of incremental kinematic and dynamic sensorimotor transformations. Exp Brain Res 164:250-259.

Kojima Y, Iwamoto Y, Yoshida K (2004) Memory of learning facilitates saccadic adaptation in the monkey. J Neurosci 25:7531-7539.

Krakauer JW, Ghez C, Ghilardi MF (2005) Adaptation to visuomotor transformations: consolidation, interference, and forgetting. J Neurosci 25:473-478.

Malfait N, Ostry DJ (2004) Is interlimb transfer of force-field adaptation a cognitive response to the sudden introduction of load? J Neurosci 24:8084-8089.

McGaugh JL (2000) Memory-a century of consolidation. Science 287:248-251.

Miall RC, Jenkinson N, Kulkarni K (2004) Adaptation to rotated visual feedback: a re-examination of motor interference. Exp Brain Res 154:201-210.

Myers DG (1998) Learning. In: Psychology, Ed 5, pp 242-267. New York: Worth.

Osu R, Hirai S, Yoshioka T, Kawato M (2004) Random presentation enables subjects to adapt to two opposing forces on the hand. Nat Neurosci 7:111-112.

Proteau L, Blandin Y, Alain C, Dorion A (1994) The effects of the amount and variability of practice on the learning of a multi-segmented motor task. Acta Psychol (Amst) 85:61-74.

Schmidt RA, Lee TL (1999) Motor control and learning, Ed 3. Champaign, IL: Human Kinetics.

Shadmehr R, Brashers-Krug T (1997) Functional stages in the formation of human long-term motor memory. J Neurosci 17:409-419.

Shadmehr R, Brandt J, Corkin S (1998) Time-dependent motor memory processes in amnesic subjects. J Neurophysiol 80:1590-1597.

Shea JB, Morgan RL (1979) Contextual interference effects on acquisition, retention, and transfer of a motor skill. J Exp Psychol Learn Mem Cogn 5:179-187.

Skinner BF (1953) Science and human behavior. New York: Macmillan.

Takahashi CD, Scheidt RA, Reinkensmeyer DJ (2001) Impedance control and internal model formation when reaching in a randomly varying dynamical environment. J Neurophysiol 86:1047-1051.

Thoroughman KA, Shadmehr R (2000) Learning of action through adaptive combination of motor primitives. Nature 407:742-747.

Wada Y, Kawabata Y, Kotosaka S, Yamamoto K, Kitazawa S, Kawato M (2003) Acquisition and contextual switching of multiple internal models for different viscous force fields. Neurosci Res 46:319-331.

Zhou W, Tao HW, Poo MM (2003) Reversal and stabilization of synaptic modifications in a developing visual system. Science 300:1953-1957. 